\title{
Mobile Phone Signals Hold Real-Time Rainfall Information
}

\author{
Techniques for monitoring rainfall with signals from mobile phone \\ networks are making their way to small-scale farmers in rural Africa.
}

\author{
By Rachel Berkowitz
}

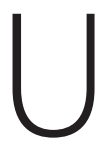
p to $70 \%$ of the food consumed in Africa is produced on 33 million small farms that rely on rainfall as their main water source. For the farmers on these lands, obtaining real-time precipitation measurements is vital for determining when to plant crops, how to apply fertilizer, and-if it is an option-how much to irrigate. Accurate rain data are also necessary for developing insurance programs that pool risk and protect farmers against poor crop yields that result from unexpected weather events.

However, many developing economies lack reliable rainfall measurements. Traditional rainfall monitoring techniques are expensive, weather stations are sparse, and satellite-based observations average over square kilometers of land-the wrong scale for farmers whose plots may be just a few hundred square meters.

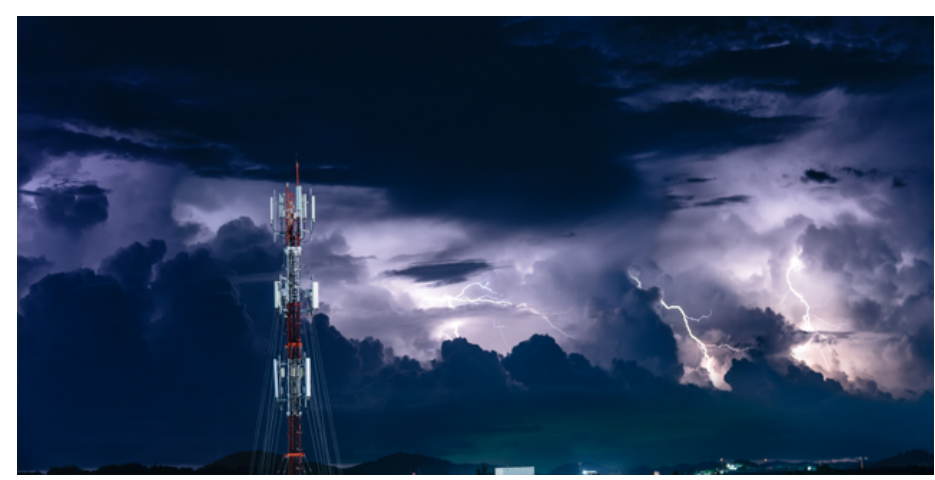

Electromagnetic waves transmitted between communication masts provide data from which atmospheric conditions can be inferred.

Credit: dima266f/stock.adobe.com
Now, thanks to extensive mobile phone infrastructure in most parts of the world, there's an effective and low-cost solution. The microwave signals beamed from one mobile phone tower to another are affected by atmospheric conditions including fog, water vapor, and most of all, rain. By measuring changes in the quality of the signals at a receiving tower, hydrologists have shown that they can derive the average rainfall along the path between the towers. Thus, each microwave "link"-or pair of connected towers in a mobile network-can serve as a real-time rain gauge along the line connecting the two towers.

Over the last decade, academic and private sector researchers have refined algorithms for converting commercial microwave link (CML) data into high-resolution rainfall maps. In developed areas of Europe and the Middle East, such maps can add valuable information to traditional monitoring and forecasting tools. But elsewhere, these maps can fill a void in weather-data collection. Researchers are now developing CML-based tools to monitor rainfall for crop production and insurance pricing in rural Africa [1]. They're building on successes in countries where the technology has been tested and adapting the methods to regions with different infrastructure constraints and different climates. They are also looking to the future, as new communication networks and smartphone technologies become more widely available.

"This innovative approach is becoming a reality in places that need it most of all, where it can help promote the economy and quality of life," says Noam David, founder of AtmosCell, an Israeli company that develops weather monitoring and forecasting software. 


\section{Into Africa}

Since the 1970s, telecommunications engineers have studied the absorption and scattering of microwave signals by rainfall and other weather conditions [2]. Raindrops are the dominant cause of signal attenuation, as their diameters roughly match the wavelengths of the gigahertz signals used for microwave links. Theoretical and experimental studies have concluded that, for any microwave frequency, the signal loss per transmission distance increases with the amount of rain along the link. Specifically, the loss is proportional to the rainfall rate raised to a certain power that depends on the signal parameters and environmental variables.

Despite its potential, rainfall monitoring with telecommunications data did not become practical until the widespread adoption of mobile phones. The breakthrough came in around 2006, with the realization that the then four million CMLs in the world could serve as rain gauges [3]. In the decade that followed, rain monitoring systems were installed in several European countries, where they provide additional meteorological data. In Africa, however, adoption has been slower.

During the monsoon season of 2012, Marielle Gosset of Geosciences Environment Toulouse in France and colleagues conducted a first-in-Africa test of rainfall monitoring based on CMLs in Burkina Faso [4]. In collaboration with the national mobile network operator Telecel Faso, Gosset's team measured attenuation in a 7-GHz signal transmitting along a 29-km-long microwave link. The CML-based system successfully detected $95 \%$ of rainy days, and the amount of rainfall that it estimated was in good agreement with measurements from a rain gauge placed in the same location.

A similar test was reported in 2019 by David from AtmosCell, Kingsley Kumah from the University of Twente in the Netherlands, and their colleagues. The team gathered signals from four CMLs that spanned several kilometers over tea farms in Kericho, Kenya. For comparison, they placed rain gauges on several farms, whose owners "were very interested in why we were measuring the data and wanted to know how they could do it themselves," Kumah says. The study showed that CMLs could record rainfall events that fell outside of the locations where rain gauges were placed.

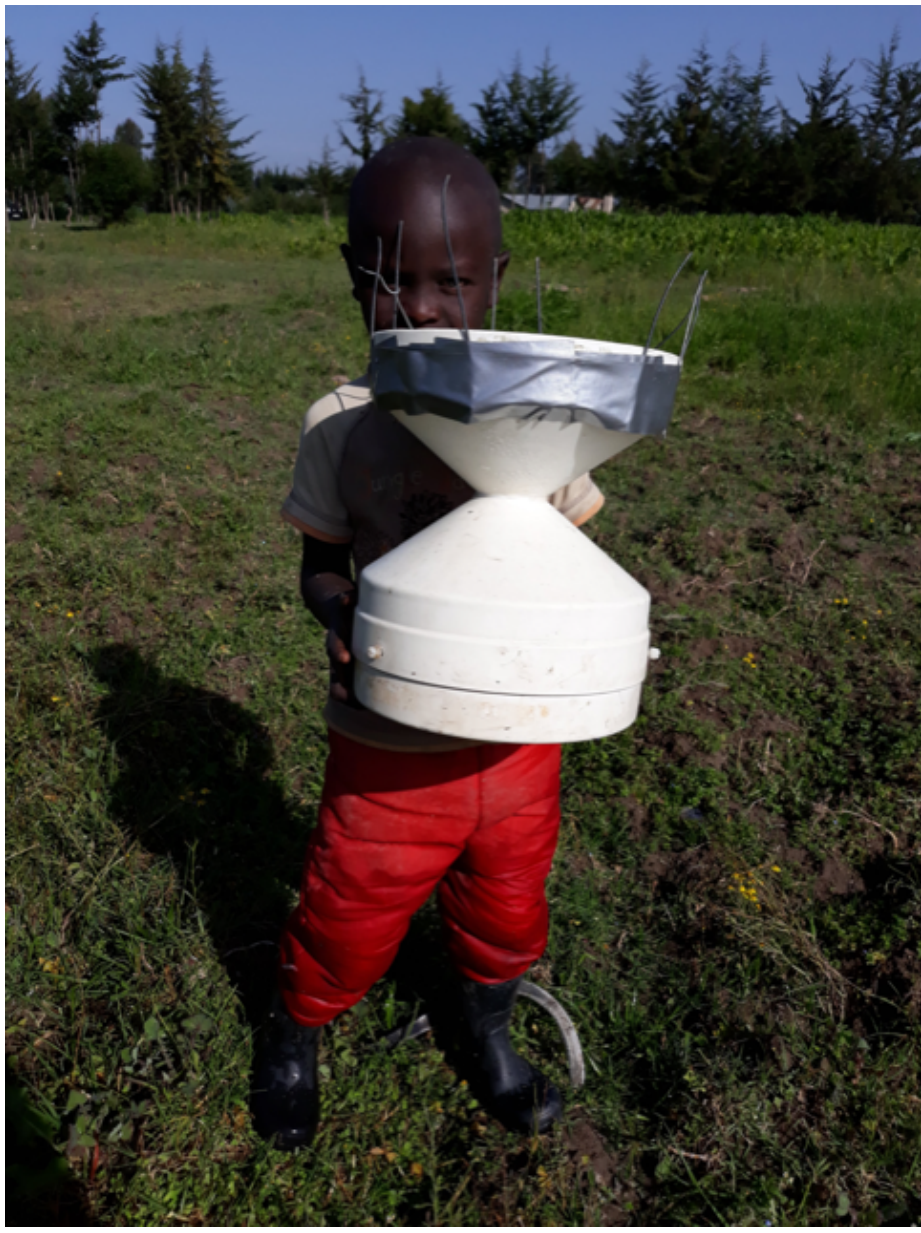

A child carries a rain gauge during its installation at a tea farm in Kericho, Kenya. Researchers from the University of Twente helped local farmers to measure rainfall rates using these tools as a way of validating the rates inferred from commercial microwave links. Credit: K. Kumah/University of Twente

In subsequent work, Kumah and colleagues performed a joint analysis of rainfall estimates from CML and from satellite data [5]. Satellites cover large areas, but they can only give an average that lacks information about specific regions. "You'd expect that if you're measuring the same thing in three ways, you'd find similar things. But that's not the case for rain gauges, satellites, and microwave links," explains Kumah. However, despite their differences, each has their utility. CML methods, in particular, can provide local detail that satellites miss while surveying more territory than a sparse network of gauges. 


\section{The Cost of Free Data}

These initial assessments showed that using CML technology enables measurements in places that have been difficult to access or where rainfall data is not routinely collected. Still, developing economies face unique challenges regarding data access, reliability, and resolution.

Decentralized networks and lack of coordination between operators means that "getting access to CML attenuation data for research and for operational application is hard," says Christian Chwala from Karlsruhe Institute of Technology in Germany, who works on CML rainfall estimation networks in Germany and Africa. With other researchers, he is seeking to improve data access through ongoing contact with the Global System for Mobile Communications Association, which represents mobile operators worldwide. Scientists and industry representatives are working together to develop a regulation procedure for unlocking data for meteorological and hydrological services, particularly in developing countries.

"Even when a telecommunications company wants to give us the data, how the data's been sampled in time is a big challenge," says Kumah. Most mobile network operators acquire and deliver data in 15-minute intervals. A rainfall event that happens in a smaller time interval may be missed. Spatial resolution is also a limiting factor for high-resolution rainfall monitoring. Link lengths tend to be longer and more sparsely distributed in rural areas than in urban ones, resulting in a higher level of uncertainty.

Remko Uijlenhoet, a hydrologist from Delft University of Technology in the Netherlands, notes that creating a 2D rainfall map from a collection of $1 \mathrm{D}$ measurements from CMLs is a challenging puzzle that requires geostatistical interpretation techniques. "It's not merely an interpolation problem," he says. The challenge is akin to creating a 3D medical image from 2D $\mathrm{x}$-ray slices.

Data reliability also varies widely. Raw signal levels may be noisy and erratic, which makes it difficult to tell if it's raining or not. "The first and most important step is to separate rainy from non-rainy periods and to infer rain rates only from the rainy periods," says Chwala. New methods for doing so draw on neural networks to help reduce false positives without increasing false-negative rates. And robust signal processing,

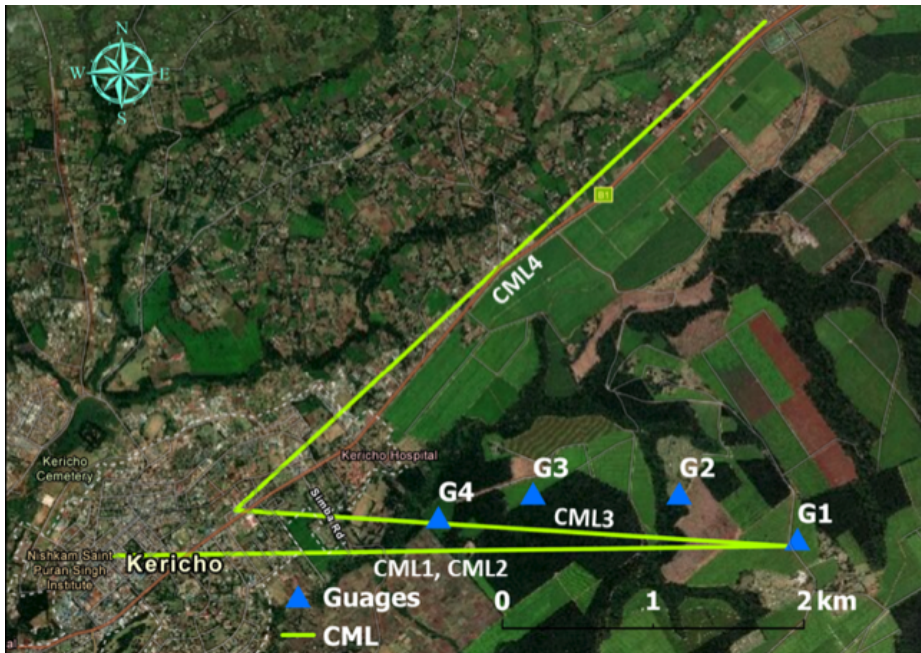

Commercial microwave links (straight lines) and rain gauges (triangles) near a farm in Kericho, Kenya.

Credit: Adapted from Ref. [1]

which includes ruling out non-rainfall sources of signal attenuation, becomes increasingly important in remote areas, where it's not possible to validate CML measurements with rain gauges and weather radar.

\section{Forecasts for the Future}

The CML landscape is evolving. Recent developments in the deployment of $5 \mathrm{G}$ networks come with new opportunities and challenges for environmental monitoring. These networks, which operate at relatively high frequencies (roughly 70-90 $\mathrm{GHz}$ ), are extremely sensitive to raindrop sizes. This sensitivity makes them good indicators of light rainfall and therefore well suited to making accurate estimates during low rainfall events, says hydrologist Martin Fencl from the Czech Technical University in Prague. Still, these new links are less likely to be important for farmers, since they are mostly deployed in urban areas.

"Ideally, in the future, local governments who allow mobile network operators to use certain radio-frequency bands for their operations could require them to share their signal-level data publicly," says Uiljenhout. This nonproprietary information could be used for the public good, he says.

Ultimately, the goal is to develop products that can be easily accessed on a smartphone. Kumah, who led the data collection 
at the Kenyan tea farm, imagines a collaboration between telecommunications companies and meteorological companies, which could provide alerts for weather events such as flash floods. "The telecom companies would provide the link signals and be at the forefront of delivering the warning to the farmers," Kumah says. For many farmers in Africa, CMLs could help determine whether the coming season will be feast or famine.

Rachel Berkowitz is a Corresponding Editor for Physics based in Vancouver, Canada.

\section{REFERENCES}

1. N. David et al., "On the power of microwave communication data to monitor rain for agricultural needs in Africa," Water 13, 730 (2021).

2. R. Uijlenhoet et al., "Opportunistic remote sensing of rainfall using microwave links from cellular communication networks," WIREs Water 5, e1289 (2018).

3. H. Messer, "Environmental monitoring by wireless communication networks," Science 312, 713 (2006).

4. A. Doumounia et al., "Rainfall monitoring based on microwave links from cellular telecommunication networks: First results from a West African test bed," Geophys. Res. Lett. 41, 6016 (2014).

5. K. K. Kumah et al., "Combining MWL and MSG SEVIRI Satellite Signals for Rainfall Detection and Estimation," Atmosphere 11, 884 (2020). 